P5 (continued)

Background: Farm to school programs have been shown to have positive impacts on child outcomes. As such, interest and initiatives in farm to ECE programs have become common. Information on ECE providers' perceptions on farm to ECE programming and current farm to ECE activities is limited.

Objectives: To describe perceived benefits, barriers, and participation of farm to ECE programming (gardening, nutrition education, and local food procurement) by ECE providers in Colorado.

Study Design, Setting, Participants: A descriptive cross-sectional design targeting ECE providers statewide was employed in 2 phases using an electronic survey that was distributed through snowball sampling: Phase 1 (46 questions) and Phase 2 (48 questions). Questions were adapted from the National Farm to School Network 2018 Farm to ECE Survey, or developed to capture specific Colorado initiatives.

Measurable Outcome/Analysis: Descriptive statistics were performed using IBM SPSS Statistics (version 25).

Results: A total of 250 complete surveys were used for analysis. Child-centered benefits were most important to respondents, and included helping children develop healthy eating habits (99\%), children connecting with nature via gardening (98\%), and opportunities for children to try more fruits/vegetables (98\%). The greatest challenges of farm to ECE programming were limited resources, including the cost of procuring local foods $(80 \%)$, and expenses and staff time associated with gardening (68\%). More than half of respondents reported that the ECE facility had a garden (59\%) and provided some form of nutrition education (57\%). Only one-third of respondents $(37 \%)$ indicated that their program procured food from local sources.

Conclusions: Findings highlight that a majority of farm to ECE activities revolve around gardening and nutrition education, with less participation in local food procurement. Future efforts to understand successes and address barriers associated with local food procurement efforts is warranted to enhance participation in all farm to ECE programming components.

Funding: None.

\section{P6 WITHDRAWN}

\section{Basic Food and Nutrition Knowledge}

\section{P7 Relationship Between Sodium Consumption and Systolic and Diastolic Blood Pressure In Adults}

Arpita Hazra, MPH, MD, Hofstra University; Jamila Conliffe, MPH, jamilaconliffe@gmail.com, Hofstra University, 1000 Hemstead Turnpike, Hempstead, NY, 11549

Background: Unhealthy eating habits is a top contributor to chronic disease burden in United States. Per American Heart Association, excessive sodium intake can raise blood pressure.

Objective: To determine correlation between sodium consumption and systolic and diastolic blood pressure (BP) in adults from 2009 to 2014.

Study Design, Setting, Participants: Cross-sectional study which examined dietary intake of sample 


\section{P7 (continued)}

population derived from National Health and Nutrition Examination Survey (NHANES). Study participants surveyed for sodium intake (mg/day) and physical examinations were conducted where systolic and diastolic BP were recorded.

Inclusion criteria included adults age $18-55$ years. Our dataset contained 29,404 participants; of these, 11,441 participants met our inclusion criteria and constitute our study sample (2,424 missing data). Amongst these, 5,529 (48.33\%) were men and 5,912 (51.67\%) were women. In our study sample, the mean age was $35.90(\mathrm{SD}=11.25)$. Our study sample constituted $16.05 \%$ Mexican Americans, 9.92\% other Hispanics, 38.60\% Non-Hispanic White, $21.70 \%$ Non-Hispanic Black, and $13.73 \%$ other Race including Multi-Racial.

Measurable Outcome/Analysis: Exposure variable is the consumption of sodium (mg per day). Outcome measure is average systolic and diastolic BP results collected from participants. Spearman Correlation along with regression analysis to quantitatively define the strength of association between sodium consumption vs systolic and diastolic BP.

Results: Mean sodium intake $3057.04 \mathrm{mg}$ (SD 1688.58, IQR 1945). Mean systolic BP $118.42 \mathrm{mmHg}$ (SD 18.36, IQR 22.0) and mean diastolic BP $65.78 \mathrm{mmHg}$ (SD 14.98, IQR 17.33). No statistically significant correlation and linear relationship found between sodium intake and systolic BP or sodium intake and diastolic BP in both men and women.

Conclusions: There is no association between dietary sodium intake and systolic BP and diastolic BP. This may be due to the size of our sample, use of medications and the fact that the sodium intake was only based on 1 day. Average daily sodium intake was noted to be higher than the recommended daily allowance of $2300 \mathrm{mg} /$ day.

Funding: None.

\section{P8 Successful Reintroduction of Landrace Orange Maize in Rural Malawi is not Dependent on Understanding Nutritional Advantages}

Aliza Stark,PhD, RD, aliza.stark@mail.huji.ac.il, The Hebrew University of Jerusalem, Robert H. Smith Faculty of Agriculture, Food and Environment, Institute of Biochemistry, Food Science and Nutrition, School of Nutritional Sciences and The International School of Agricultural Sciences, PO Box 12, Rehovot, ISRAEL, 76100; Alex Katola, MS, The Hebrew University of Jerusalem, Robert H. Smith Faculty of Agriculture, Food and Environment, Institute of Biochemistry, Food Science and Nutrition, School of Nutritional Sciences, Rehovot, ISRAEL; Victoria Ndolo, PhD, University of Malawi, Chancellor College, Zomba, Malawi; David Tembo, PhD, University of Malawi, The Polytechnic, Blantyre, Malawi; Mangani Katundu, $P h D$, University of Malawi, Chancellor College, Zomba, Malawi

Background: Orange maize, rich in carotenoids, is a possible sustainable solution for combating Vitamin A deficiency and reducing dependence on fortification. The
ProFarmer project in Malawi has successfully re-introduced landrace orange maize (MW5021) to replace or complement white hybrid varieties in over 2000 farms.

Objectives: This study was designed to determine the acceptability of foods made with orange maize in children and to assess the knowledge, attitudes and behaviors (KAB) of women in farming families that currently grow orange maize.

Study Design, Setting, Participants: Organoleptic evaluation of white and orange maize food products was carried out in school age children $(n=160)$ of ProFarmer families living in the Dedza and Thyolo Districts. Additionally, a cross sectional study was completed in female household members $(n=336)$ living on farms that produce orange maize. Participants were interviewed regarding reintroduction of orange maize and diet quality was assessed.

Measurable Outcome/Analysis: Acceptability of orange maize food products was determined using a 7point hedonic scale. Women's responses to questionnaires were used to determine nutritional knowledge regarding orange maize. A Minimum Dietary Diversity questionnaire (MDD-W) evaluated diet adequacy. Independent $t$ tests and Chi square tests were performed where appropriate.

Results: Organoleptic testing of orange maize porridges by children indicated high levels of acceptance. Survey results showed positive attitudes towards the orange maize with $75-85 \%$ of households consuming the product. However, only $32 \%$ of the women understood its nutritional benefits. Dietary Diversity scores, a proxy for nutrient adequacy, were low with only $34 \%$ of women consuming a sufficiently diverse diet.

Conclusions: Despite low levels of nutritional knowledge, landrace orange maize is well accepted by both children and adults in rural Malawi and is an option for increasing dietary sources of provitamin A. Nutrition education emphasizing the advantages of eating carotenoidrich foods and expanding diet diversity are recommended. Funding: Pears Foundation, UK; The International School of Agricultural Sciences - Hebrew University

\section{P9 Exploring Parental Beliefs and Practices Towards Sugar-Sweetened Beverage Intake in Young Children}

Christen Cupples Cooper,EdD, RDN, ccooper3@pace.edu, Pace University, College of Health Professions, 861 Bedford Rd, L23, Pleasantville, NY, 10570; Angela Northrup, $P h D, R N, F N P$, Pace University, College of Health Professions; Michelle Iannacchino, BS, Pace University, College of Health Professions

Background: High sugar-sweetened beverage (SSB) intake during childhood is a major risk factor for obesity and other adverse health outcomes. SSB include drinks with added sugar such as soda, fruit and sports drinks, flavored teas, and milk. SSB are high-calorie, low-nutrient, and major sources of added sugar. Nearly half (47\%) of 